\title{
Examining Social Capital in Relation to Sleep Duration, Insomnia, and Daytime Sleepiness
}

\section{ABSTRACT}

\section{Objective}

Sleep, which plays an important role in health and well-being, is socially patterned such that certain demographic groups have worse sleep health than others. One possible mechanism driving sleep disparities is social capital. The current study examines the association between social capital and self-reported sleep variables (e.g., duration, insomnia symptoms, and daytime sleepiness) among a sample of 1,007 participants from the Sleep Health and Activity, Diet and Environment Study (SHADES).

Logistic regressions were used to estimate whether the sleep variables were associated with social capital measures. All models control for age, sex, race/ethnicity (Non-Hispanic White, Black/African-American, Hispanic/Latino, Asian, and multicultural/other), income, and education (less than high school, high school graduate, some college, and college graduate).

\section{Results}

Lower likelihood of membership in groups was seen for long sleepers (>9hrs, p-value<.05) and beliefs that neighbors rarely/never help each other was more likely among short sleepers (5-6hrs, p-value<.05), relative to 7-8 hour sleepers. A decreased sense of belonging was seen among short sleepers (5-6hrs, p-value<.05). Decreased likelihood of trust was reported by those with moderate-severe insomnia ( $p$-value<.05). Similarly, neighborhood improvement efforts were less likely among individuals with moderate-tosevere insomnia ( $p$-value $<.05)$.

\section{Conclusions}

31 Results of our study show that short and long sleep duration, as well as insomnia, were inversely related to measures of social capital, such as group memberships and a sense of neighborhood belonging. Future research may explore the directionality of the relationship between social capital and sleep and perhaps consider future interventions to improve low social capital and/or poor sleep in community samples. 


\section{INTRODUCTION}

3 Sleep plays an important role in health and well-being. ${ }^{1-3}$ Poor sleep health (e.g., sleep

4 duration for less than or equal to 6 hours of sleep or poor sleep quality) is associated

5 with poor mental health, anxiety, and depression;,4, higher risk of hypertension and

6 cardiovascular disease $;^{6-8}$ and greater mortality risk. ${ }^{9,10}$ Unfortunately, worse sleep

7 health has been shown to be more common among individuals from low socioeconomic

8 status (SES, low income and education) compared to other groups. ${ }^{11-15}$ This paper

9 contributes to a growing body of research on neighborhood-level characteristics and

10 their relationship to sleep. ${ }^{16-19}$ Specifically, we examine social capital, a social network

11 concept referring to the quality of connections among individuals in a community, ${ }^{20-23}$

12 and its relationship to several dimensions of sleep health, including sleep duration,

13 insomnia, and daytime sleepiness.

15 Previous research has examined a range of neighborhood factors and their association

16 to sleep. Research has shown high risk for sleep apnea among children living in

17 communities that have low average SES. ${ }^{18,19}$ After adjustment for SES, research has

18 found favorable neighborhood factors (lower neighborhood disorder and higher safety

19 and social cohesion) are associated with higher objectively measured sleep duration, ${ }^{16}$

20 and undesirable neighborhood factors (higher neighborhood disorder and lower safety

21 and social cohesion) are associated with shorter sleep duration. ${ }^{17}$ Similarly, research

22 has found, controlling for SES, low social cohesion is associated with shorter sleep

23 duration. ${ }^{24}$ Also, research has found an association between unfavorable neighborhood 
1 factors (crime, noise, and cleanliness) and poor physical health is partially mediated by

2 sleep quality. ${ }^{25}$ Although previous literature has demonstrated an association between

3 sleep and neighborhood factors, previous studies have not fully addressed the

4 directionality of the relationships among specific social capital components and sleep

5 health dimensions. Further, this growing body of literature to suggest neighborhood

6 factors matter for sleep outcomes, ${ }^{16-19}$ little attention has been paid to social

7 capital. ${ }^{17,26,27}$ Although our research is cross-sectional in nature, we examine the

8 relationships among specific social capital and sleep health dimensions.

10 Social capital refers to the nature and quality of social relationships and connections

11 among individuals in a neighborhood or social network. Also, in addition to the nature of

12 these relationships, social capital has to also do with the resources a person may

13 receive by virtue of their social connections. Social network variables such as social

14 capital are important to consider as evidence shows that health behaviors such as

15 alcohol consumption, smoking, ${ }^{29,30}$ and sleep duration, ${ }^{31}$ are remarkably homogenous

16 among groups of socially connected individuals. ${ }^{28}$ Previous research has found low

17 social capital is associated with depression ${ }^{32,33}$ and mortality risk, ${ }^{34}$ whereas high social

18 capital is associated with physiological well-being. ${ }^{35}$ Research has also shown that,

19 after controlling for SES, social capital is associated with self-reported health. ${ }^{36}$ With

20 regards to sleep, one study was identified that examined social capital among

21 community-dwelling Japanese individuals, finding poor social capital is associated with

22 insufficient sleep duration. ${ }^{37}$ 
1 The Social-Ecological Model of Sleep Health ${ }^{9,38}$ posits that poor sleep health is driven

2 by social-contextual factors that underpin health broadly and sleep health specifically.

3 Obtaining a better understanding of relationships between sleep health and social

4 capital may allow for a deeper understanding of the social-environmental causes and/or

5 consequences of poor sleep health. As such, the goal of this paper is to examine

6 discrete measures of social capital and their relationship to a range of sleep health

7 parameters (sleep duration, insomnia, and daytime sleepiness).

9 Social capital is operationalized in terms of the following dimensions: 1) participation in

10 social or community organization(s) (i.e., the number of formal social groups, such as

11 clubs, a person belongs to), 2) neighborhood helping behavior (i.e., the degree to which

12 neighbors help each other), 3) neighborhood belonging (i.e., the degree to which

13 individuals feel like their neighborhood makes them feel welcome and that they belong),

14 4) neighborhood trust (i.e., the degree to which neighbors trust each other), and 5)

15 neighborhood improvement (i.e., the degree to which neighbors work together to

16 improve the neighborhood). ${ }^{23,39,40}$ Based on previous findings that demonstrate an

17 inverse association between sleep duration and social capital, ${ }^{37}$ we extend these

18 findings to hypothesize an inverse association between social capital and three

19 dimensions of sleep health, including: duration, insomnia, and sleepiness. Consistent

20 with prior research examining neighborhood factors, ${ }^{16,17,24,37}$ we propose to control for

21 SES so as to focus on the association between social capital and sleep health. Using

22 cross-sectional data from the Sleep Health and Activity, Diet and Environment Study

23 (SHADES) survey of adults, we examine following hypothesized associations: 
1

\section{Data Source}

18 We analyzed data from the Sleep and Healthy Activity, Diet, Environment, and

19 Socialization (SHADES) study. SHADES was a community-level study of adults

$20(\mathrm{~N}=1007)$ age $22-60$ in the Philadelphia area, including Philadelphia County and the

21 neighboring Delaware, Montgomery, Bucks, and Chester Counties. Respondents were

22 recruited through advertisements posted in various public spaces and community

23 centers. The study included a survey that was completed online or in person between 
1 January 2013 and October 2014. There was only one assessment and no follow-ups,

2 and consisted of a number of items assessing sleep, health, behavior, and

3 environmental factors. Because all online surveys were validated and all in-person

4 surveys ensured completion, there was no missing data.

6 Measures

7 Sleep duration was measured using one of the sleep questions from the National Health

8 and Nutrition Examination Survey (NHANES): 41,42 "How much sleep do you usually get

9 at night on weekdays or workdays?" Responses were recorded in whole numbers and

10 categorized as very short ( $\leq 4$ hours), short (5-6 hours), recommended duration (7-8

11 hours) and long ( $\geq 9$ hours), as is consistent with previous studies. $8,43-45$

13 Insomnia symptoms were measured using the Insomnia Severity Index (ISI). ${ }^{46}$

14 Accordingly, ISI scores range from 0 to 28 , with higher scores indicating greater severity

15 of insomnia symptoms. Recommended cutoffs include 0 to 7 for no insomnia, 8 to 14 for

16 mild insomnia, 15 to 21 for moderate insomnia and 22 to 28 for severe insomnia. Due to

17 the low frequency of severe insomnia, the final two categories were collapsed into a

18 moderate-severe category. The ISI is routinely used as an outcome measure for

19 insomnia research ${ }^{47}$ and is well-validated for research. ${ }^{48}$

20

21 Daytime sleepiness was measured using the Epworth Sleepiness Scale (ESS). This 8-

22 item instrument is routinely used in research ${ }^{49,50}$ and is well-validated. ${ }^{51}$ Participants

23 were asked to indicate the likelihood that they would be unable to maintain wakefulness 
1 in a variety of situations, including sitting and reading, watching TV, and sitting inactive

2 in a public place, etc. Responses are summed and scores can range from 0 to 24 .

3 Recommended cutoffs for sleepiness include 0 to 9 for mild sleepiness and 10 or higher

4 for severe daytime sleepiness. ${ }^{51}$

6 Social capital was measured using variables consistent with prior research. ${ }^{32,52,53}$ Social

7 capital domains assessed included group memberships, neighborhood helping

8 behavior, and neighborhood belonging, trust, and improvement. Group memberships

9 were assessed with the questions "how many local groups or organizations in your

10 neighborhood do you currently participate in such as social, political, religious, school-

11 related, or athletic organizations?" Responses were categorized as 0,1 , or $\geq 2$ or more

12 memberships. Neighborhood helping behavior was assessed with the question, "Please

13 rate how likely people in your neighborhood are willing to help their neighbors with

14 routine activities such as picking up their trash cans, or helping to shovel snow. Would

15 you say that most people in your neighborhood are always, often, sometimes, rarely, or

16 never willing to help their neighbors?" Responses were coded as "Never," "Rarely,"

17 "Sometimes," "Often," and "Always." Due to cell sizes at the extremes, responses were

18 collapsed to "Never/Rarely," "Sometimes," and "Often/Always." Neighborhood belonging

19 and trust were assessed by asking participants to agree or disagree with the following

20 statements: "I feel that I belong and am a part of my neighborhood," and "Most people in

21 my neighborhood can be trusted." These were coded as "Agree" vs "Disagree." Finally,

22 neighborhood improvement was assessed by asking participants, "Have people in your

23 neighborhood ever worked together to improve the neighborhood? For example, 
1 through a neighborhood watch, creating a community garden, building a community

2 playground, or participating in a block party." Responses were categorized as "Yes" or

3 "No." For purposes of analysis, the discreet measures of social capital are maintained in

4 the current study (e.g., group memberships).

6 Covariates included age, sex, race/ethnicity (Non-Hispanic White, Black/African-

7 American, Hispanic/Latino, Asian, and multicultural/other), education (less than high

8 school, high school graduate, some college, and college graduate), and income. These

9 were chosen because previous studies have shown them to be related to sleep duration 10 and quality, ${ }^{54-56}$ and social functioning. $.57,58$

12 Statistical Analyses

13 Analysis of variance (ANOVA) was conducted to examine differences in demographic

14 factors by social capital. A series of binomial and multinomial logistic regression models

15 for sleep variables and each of the five social connectedness variables were assessed.

16 In each case, the social capital variable was the outcome and the sleep variable was

17 the predictor. In addition to separate models for each sleep variable (adjusted for

18 covariates), combined models included all sleep variables simultaneously. The reason

19 for including this combined model is that many of the sleep variables overlap with each

20 other and, therefore, associations may be due to the overlapping issue of "poor sleep"

21 and not the specific sleep aspect assessed (i.e., sleep duration, insomnia symptoms, or

22 sleepiness). Therefore, a combined model examines whether any individual sleep

23 variable explains variance in the social capital variables that is unique, after controlling 
1 for the other sleep variables. In this way, we can examine whether there is a

2 relationship with insomnia that is distinct the other sleep variables. The $95 \%$ confidence

3 intervals (Cls) were computed. Analyses were performed using STATA software version

414 (StataCorp, College Station, TX, USA). All statistical tests were two-tailed. Statistical

5 significance was set at the $P<0.05$ level.

6

\section{RESULTS}

8 Characteristics of the Sample

9 Among participants, average age was 34.0 years (s.d. $=9.4$ years) and the sample was

$1050.9 \%$ male. Participants were $58.1 \%$ non-Hispanic white, $55.9 \%$ reported a college

11 degree, and $18.6 \%$ reported income of $\$ 75,000$ or higher. Among participants,

12 recommended duration (7-8 hours) was reported by $47.7 \%$. Approximately one third of

13 participants reported no insomnia symptoms (34.8\%). Daytime sleepiness scores

14 averaged $7.8(\mathrm{sd}=4.7)$. See Table 1 for a detailed summary of the study sample and

15 sleep variables.

16

17 Participation in Social or Community Groups

18 In the separate models, very short ( $\leq 4$ hours) and long sleepers ( $>9$ hours) were

19 respectively approximately half as likely as $7-8$ hour sleepers to report membership in a

20 social or community organization. In the combined model, only the relationship between

21 long sleep and membership in social or community organizations was significant, so

22 that long sleepers were half as likely as $7-8$ hour sleepers to report participation in one 
1 social or community organization. No statistically significant association was identified

2 for short sleep duration, insomnia, or sleepiness (no support for $\mathrm{H} 1$, see Table 2).

$4 \quad$ Helping Neighbors

5 In the separate models, very short sleepers ( $\leq 4$ hours) and short (5-6 hours) sleepers

6 were approximately 1.5 times more likely to report rarely or never helping their

7 neighbors, compared to 7-8 hour sleepers. In addition, individuals with moderate-severe

8 insomnia and daytime sleepiness were also about 1.5 times more likely to report rarely

9 or never helping their neighbors. In the combined model, only short sleep (5-6 hours)

10 was significant, so that short sleepers were approximately 1.5 times more likely to report

11 rarely never helping behavior (no support for $\mathrm{H} 2$, see Table 3 ).

13 Sense of Belonging, Neighborhood Trust and Improving the Neighborhood

14 In separate models, short sleepers (5-6 hours), individuals with moderate-to-severe

15 insomnia and high daytime sleepiness were less likely to report neighborhood belonging

16 than 7-8 hour sleepers. In the combined model, only short sleepers were significantly

17 less likely to report a sense of neighborhood belonging (no support for $\mathrm{H} 3$ ). In separate

18 and combined models, individuals with moderate-to-severe insomnia were less likely to

19 report neighborhood trust (no support for $\mathrm{H} 4$ ). In separate models, short sleepers and

20 individuals reporting moderate-to-severe insomnia were significantly less likely to report

21 neighborhood improvement behaviors. In combined models, individuals with moderate-

22 to-severe insomnia were significantly less likely to report improvement (no support for

$23 \mathrm{H}$, see Table 4). 


\section{DISCUSSION}

4 There is a large and growing body of literature linking social and environmental factors,

5 such as social capital, with individual health outcomes. ${ }^{36,52,53}$ While many areas of

6 health have been examined, ${ }^{32-35}$ we know less about the relationship between social

7 capital and sleep health. Due to the health disparities in sleep and population health

8 broadly, it is vital to identify barriers to sleep health, such as poor social capital, in order

9 to advance population health for all. ${ }^{3,59}$ This paper extends social capital research to

10 examine the relationship between several social capital variables (e.g., group

11 participation, neighborhood belonging) and sleep-related variables, including sleep

12 duration, insomnia and sleepiness. We do so in a cross-sectional study among adults

13 living in Philadelphia and surrounding counties.

15 Our findings contribute to the growing literature on social capital and sleep health. ${ }^{16-19}$

16 Our results show that long sleepers were less likely to report participation in social or

17 community organizations in both separate and combined models, but not short

18 sleepers. This is a novel contribution to the literature, as Win and colleagues found an

19 association between short sleep duration and poor social capital, ${ }^{37}$ and Pabayo and

20 colleagues found an association between a similar construct, social fragmentation, and

21 short sleep duration. ${ }^{24}$ Then, consistent with the work from Win and Pabayo, our results

22 found short sleepers were less likely to report helping behaviors and less likely to report

23 a sense of belonging in their community, in both separate and combined models. 
2 With regards to sleep duration, there is an argued U-shaped relationship whereby short

3 sleep (less than 7 hours) is associated with health risks, as is long sleep (greater than 9

4 hours). ${ }^{60}$ While the health detriment associated with short sleeping is well-

5 documented, ${ }^{6,7,9,56}$ there is less consensus regarding long sleep and its health

6 detriment. ${ }^{2,61}$ Our results show long sleepers are less likely to report social capital (e.g.,

7 group memberships) compared to those sleeping 7 to 8 hours, which is a finding that

8 buttresses the U-shaped curve argument that long sleep is associated with adverse

9 outcomes. However, one argument to explain the association between long sleep and

10 poor social capital in this study could be that long sleep may be due to conditions such

11 as anxiety or depression, which would lower one's capacity and motivation for group

12 interaction and its social capital benefits. On the other hand, it may be that long

13 sleepers have less total available time in a 24-hour cycle for socializing thereby limiting

14 their ability to fully engage in their social network.

16 While other studies examined sleep duration and quality as it relates to social capital,

17 cohesion, and neighborhood-related factors, ${ }^{17,24,27,37}$ few have examined daytime

18 sleepiness and insomnia as they relate to social capital. Results from our study found

19 insomnia was significantly, inversely associated with neighborhood trust and

20 improvement behavior in combined models. This is a novel finding, as previous work

21 found a significant relationship between social cohesion and sleep duration and sleep

22 quality, but not insomnia. ${ }^{17,24,27,37}$ Further, daytime sleepiness, although a predictor in

23 separate models of low neighborhood helping behavior and low neighborhood 
1 belonging, was not a significant predictor of either social capital measure in combined

2 models.

4 It is interesting to note the different patterns of directionality observed in separate

5 models compared to patterns observed in combined models predicting sleep health

6 dimensions and social capital factors. For instance, daytime sleepiness was a

7 significant predictor of social capital in separate models but not in combined models.

8 Our aim in analyzing sleep health variables together in combined models was to

9 examine whether any specific sleep-related variable demonstrated an association that

10 was unique and not otherwise explained by the others. In this way, the lack of significant

11 association for sleepiness in combined models suggests that the variance explained by

12 sleepiness generally overlaps with that explained by other sleep variables.

14 Although not all associations between sleep and social capital components were

15 significant in both separate and combined models, there is a general trend toward a

16 relationship between poor sleep health (e.g., short or long sleep, moderate-to-severe

17 insomnia) and poor social capital (e.g., fewer group memberships, less neighborhood

18 helping behavior, belonging, trust, and improvement). It could be that certain

19 dimensions of poor sleep health (e.g., short sleep) have specific implications for aspects

20 of social behavior, but not others. Combining sleep health dimensions (e.g., duration

21 and insomnia) into a single scale may be one opportunity for future research. 
1 In addition, future research might explore the causal direction between sleep duration,

2 insomnia, and sleepiness and social capital in longitudinal research to examine how

3 sleep health and social capital evolve over time. It would also be insightful to examine

4 the impact of improvements to social capital and their implications for sleep health, or

5 conversely, improvements to social capital and their benefit for sleep health.

6

7 In sum, social capital may be a concept with potential for advancing our understanding

8 of the link between social/environmental factors, sleep, and perhaps also population

9 health. For instance, poor social capital and lower access to resources (e.g., both

10 tangible such as goods or financial assistance and intangible, such as social support)

11 may be a driving force in the link between short or long sleep and undesirable health

12 outcomes.

\section{Limitations}

15 The sleep-reported data in this study are retrospective self-report and possible biases

16 exist in reports of sleep duration, insomnia, and sleepiness. In addition, the data were

17 collected in one geographical location and in predominantly urban and suburban

18 settings. Data collected from alternative locations may yield different results. Also, the

19 cross-sectional nature of the study precludes any causality inferences. It should be

20 noted that the current study collected self-reported sleep, as opposed to actual sleep

21 need. Also, while our paper is the first to our knowledge to offer a comprehensive

22 assessment of various social capital dimensions and sleep health parameters, our data

23 are cross-sectional. Future research is needed to understand the directionality of the 
1 relationship between social capital and sleep health. Finally, SES and neighborhood

2 characteristics, such as social cohesion, have been conceptualized in the literature as

3 similar constructs, ${ }^{18,19}$ yet research examining specific neighborhood dimensions, such

4 as social fragmentation and social capital, have controlled for aspects of SES in their

5 analyses. ${ }^{17,24,27,37}$ Consistent with this approach, our analysis controlled for aspects of

6 SES so as to specifically examine social capital. Thus, our findings may be different if

7 we did not control for SES in our study. Future research may examine the conceptual

8 overlap between specific SES dimensions (e.g., income, education, race) and their

9 relationship to social capital.

\section{Implications and Future Directions}

13 Results from the current study offer several directions for future research. We detected

14 an association between long sleep and several social capital. These patterns may be

15 further explored in future research, as the previous evidence suggests an association

16 between short sleep and poor social capital, research in the future may examine

17 longitudinal patterns of sleep health and social capital to understand the directionality of

18 the association between these constructs.

20 Public health approaches may be considered for methods, such as engaging

21 community health workers or community events that specifically aim to improve social

22 capital building events and messaging, so as to increase social capital among

23 individuals who reside in communities at risk for poor social capital and poor sleep 
1 health or consider community-based methods for improving sleep health as a method

2 for improving social capital. Also, as sleep is a behavior that is linked to a suite of health

3 outcomes, including chronic conditions, such as diabetes, hypertension, and mortality

4 risk, ${ }^{6,9,44}$ it may be interesting to examine how improving social capital may improve

5 sleep and consequently, chronic disease management. Finally, future research may

6 combine social network analysis (e.g., examining connections among individuals) with

7 measures of social capital to examine how these factors interact and bear consequence

8 for sleep and health.

9

10 Conclusion

11 Our study offers preliminary evidence for the link between social capital and several

12 self-reported sleep variables. Specifically, we find preliminary evidence to suggest poor

13 sleep health (e.g., short and long sleep duration and insomnia) is associated with poor

14 social capital ( e.g., less participation in social or community organizations, lower

15 helping behavior and less neighborhood trust, belonging, and improvement). The

16 importance of social network factors, such as social capital, for health has been

17 demonstrated in other domains, including smoking and alcohol consumption. ${ }^{29,30}$ Social

18 capital may be a social network factor that helps advance our understanding of the link

19 between neighborhoods, sleep, and population health. 
4. Hyyppä MT, Kronholm E, Mattlar CE. Mental well-being of good sleepers in a random population sample. Br J Med Psychol. 1991;64 ( Pt 1):25-34.

5. Calkins AW, Hearon BA, Capozzoli MC, Otto MW. Psychosocial predictors of sleep dysfunction: the role of anxiety sensitivity, dysfunctional beliefs, and neuroticism. Behav Sleep Med. 2013;11(2):133-143.

6. Buxton OM, Marcelli E. Short and long sleep are positively associated with obesity, diabetes, hypertension, and cardiovascular disease among adults in the United States. Soc Sci Med. 2010;71(5):1027-1036.

7. Gangwisch JE, Heymsfield SB, Boden-Albala B, et al. Short sleep duration as a risk factor for hypertension. Hypertension. 2006;47(5):833-839.

8. Zizi F, Jean-Louis G, Brown CD, Ogedegbe G, Boutin-Foster C, McFarlane SI. Sleep duration and the risk of diabetes mellitus: epidemiologic evidence and pathophysiologic insights. Curr Diab Rep. 2010;10(1):43-47.

9. Grandner MA, Hale L, Moore M, Patel NP. Mortality associated with short sleep duration: The evidence, the possible mechanisms, and the future. Sleep Med Rev. 2010;14(3):191-203. doi:10.1016/j.smrv.2009.07.006

10. Patel SR, Ayas NT, Malhotra MR, et al. A prospective study of sleep duration and mortality risk in women. Sleep. 2004;27(3):440-444.

11. Hale L, Peppard PE, Young T. Does the demography of sleep contribute to health disparities. Sleep Disord Their Impact Public Health. 2007:1-17.

12. Jackson CL, Hu FB, Redline S, Williams DR, Mattei J, Kawachi I. Racial/ethnic disparities in short sleep duration by occupation: The contribution of immigrant status. Soc Sci Med. 2014;118:71-79. 
13. Nunes J, Jean-Louis G, Zizi F, et al. Sleep duration among black and white Americans: results of the National Health Interview Survey. J Natl Med Assoc. 2008;100(3):317-322.

14. Grandner MA, Williams NJ, Knutson KL, Roberts D, Jean-Louis G. Sleep disparity, race/ethnicity, and socioeconomic position. Sleep Med. 2016;18:7-18.

15. Pandey A, Donat M, Mahmud S, Brimah P, Zizi F, Jean-Louis G. Rlsk of obstructive sleep apnea and anxiety among blacks with metabolic syndrome. Chest. 2011;140(4_MeetingAbstracts):796A-796A. doi:10.1378/chest.1113865

16. Johnson DA, Simonelli G, Moore K, et al. The Neighborhood Social Environment and Objective Measures of Sleep in the Multi-Ethnic Study of Atherosclerosis. Sleep. September 2016.

17. Desantis AS, Diez Roux AV, Moore K, Baron KG, Mujahid MS, Nieto FJ. Associations of neighborhood characteristics with sleep timing and quality: the Multi-Ethnic Study Of Atherosclerosis. Sleep. 2013;36(10):1543-1551. doi:10.5665/sleep.3054

18. Brouillette RT, Horwood L, Constantin E, Brown K, Ross NA. Childhood sleep apnea and neighborhood disadvantage. J Pediatr. 2011;158(5):789-795.e1. doi:10.1016/j.jpeds.2010.10.036

19. Spilsbury JC, Storfer-Isser A, Kirchner HL, et al. Neighborhood disadvantage as a risk factor for pediatric obstructive sleep apnea. J Pediatr. 2006;149(3):342-347. doi:10.1016/j.jpeds.2006.04.061

20. Bourdieu P. La Distinction: Critique Sociale Du Jugement. Vol 12. Editions de minuit; 1979.

21. Burt RS. A note on social capital and network content. Soc Netw. 1997;19(4):355373.

22. Coleman JS. Social capital in the creation of human capital. Am J Sociol. 1988;94:S95-S120.

23. Hawe P, Shiell A. Social capital and health promotion: a review. Soc Sci Med. 2000;51(6):871-885. doi:10.1016/S0277-9536(00)00067-8

24. Pabayo R, Molnar BE, Street N, Kawachi I. The relationship between social fragmentation and sleep among adolescents living in Boston, Massachusetts. $J$ Public Health. 2014;36(4):587-598. doi:10.1093/pubmed/fdu001

25. Hale L, Hill TD, Burdette AM. Does sleep quality mediate the association between neighborhood disorder and self-rated physical health? Prev Med. 2010;51(34):275-278. doi:10.1016/j.ypmed.2010.06.017 
1 26. Faubel R, Lopez-Garcia E, Guallar-Castillón P, et al. Sleep duration and healthrelated quality of life among older adults: a population-based cohort in Spain. Sleep. 2009;32(8):1059.

27. Johnson AL, Jung L, Song Y, Brown KC, Weaver MT, Richards KC. Sleep deprivation and error in nurses who work the night shift. $J$ Nurs Adm. 2014;44(1):17-22. doi:10.1097/NNA.0000000000000016

28. McPherson M, Smith-Lovin L, Cook JM. Birds of a feather: Homophily in social networks. Annu Rev Sociol. 2001;27:415-444.

29. Christakis NA, Fowler $\mathrm{JH}$. The collective dynamics of smoking in a large social network. N Engl J Med. 2008;358(21):2249-2258.

30. Rosenquist JN, Murabito J, Fowler JH, Christakis NA. The spread of alcohol consumption behavior in a large social network. Ann Intern Med. 2010;152(7):426.

31. Mednick SC, Christakis NA, Fowler JH. The spread of sleep loss influences drug use in adolescent social networks. PloS One. 2010;5(3):e9775.

32. Economou M, Madianos M, Peppou LE, Souliotis K, Patelakis A, Stefanis C. Cognitive social capital and mental illness during economic crisis: A nationwide population-based study in Greece. Soc Sci Med. 2014;100:141-147. doi:10.1016/j.socscimed.2013.11.006

33. Landstedt E, Almquist YB, Eriksson M, Hammarström A. Disentangling the directions of associations between structural social capital and mental health: Longitudinal analyses of gender, civic engagement and depressive symptoms. Soc Sci Med. 2016;163:135-143. doi:10.1016/j.socscimed.2016.07.005

34. Kawachi I, Kennedy BP, Lochner K, Prothrow-Stith D. Social capital, income inequality, and mortality. Am J Public Health. 1997;87(9):1491-1498.

35. Lindström M, Giordano GN. The 2008 financial crisis: Changes in social capital and its association with psychological wellbeing in the United Kingdom - A panel study. Soc Sci Med. 2016;153:71-80. doi:10.1016/j.socscimed.2016.02.008

36. Kawachi I, Kennedy B, Glass R. Social capital and self-rated health: a contextual analysis. Am J Public Health. 1999;89(8):1187-1193.

37. Win T, Yamazaki T, Kanda K, Tajima K, Sokejima S. Neighborhood social capital and sleep duration: a population based cross-sectional study in a rural Japanese town. BMC Public Health. 2018;18. doi:10.1186/s12889-018-5204-4

38. Grandner MA. Sleep, health, and society. Sleep Med Clin. 2017;12(1):1-22. 
39. Chen D, Yang T-C. The pathways from perceived discrimination to self-rated health: An investigation of the roles of distrust, social capital, and health behaviors. Soc Sci Med. 2014;104:64-73. doi:10.1016/j.socscimed.2013.12.021

40. De Clercq B, Pfoertner T-K, Elgar FJ, Hublet A, Maes L. Social capital and adolescent smoking in schools and communities: A cross-classified multilevel analysis. Soc Sci Med. 2014;119:81-87. doi:10.1016/j.socscimed.2014.08.018

41. Centers for Disease Control and Prevention. National Health and Nutrition Examination Survey Phone Follow-up Dietary Interviewer Procedures Manual.; 2008.

42. National Center for Health Statistics. Analytic and reporting guidelines: the national health and nutrition examination survey (NHANES). Hyattsville MD Cent Dis Control Prev. 2006.

43. Gangwisch JE, Malaspina D, Boden-Albala B, Heymsfield SB. Inadequate sleep as a risk factor for obesity: analyses of the NHANES I. SLEEP. 2005;28(10):1289.

44. Grandner MA, Chakravorty S, Perlis ML, Oliver L, Gurubhagavatula I. Habitual sleep duration associated with self-reported and objectively determined cardiometabolic risk factors. Sleep Med. 2014;15(1):42-50.

45. Jean-Louis G, Williams NJ, Sarpong $D$, et al. Associations between inadequate sleep and obesity in the US adult population: analysis of the national health interview survey (1977-2009). BMC Public Health. 2014;14(1):1.

46. Bastien $\mathrm{CH}$, Vallières $\mathrm{A}$, Morin $\mathrm{CM}$. Validation of the Insomnia Severity Index as an outcome measure for insomnia research. Sleep Med. 2001;2(4):297-307.

47. Morin CM, Belleville G, Bélanger L, Ivers $\mathrm{H}$. The Insomnia Severity Index: psychometric indicators to detect insomnia cases and evaluate treatment response. Sleep. 2011;34(5):601-608.

48. Chaudhary NS, Kampman KM, Kranzler HR, Grandner MA, Debbarma S, Chakravorty S. Insomnia in alcohol dependent subjects is associated with greater psychosocial problem severity. Addict Behav. 2015;50:165-172.

49. Buysse DJ, Hall ML, Strollo PJ, et al. Relationships between the Pittsburgh Sleep Quality Index (PSQI), Epworth Sleepiness Scale (ESS), and clinical/polysomnographic measures in a community sample. J Clin Sleep Med JCSM Off Publ Am Acad Sleep Med. 2008;4(6):563-571.

50. Gradisar M, Gardner G, Dohnt H. Recent worldwide sleep patterns and problems during adolescence: a review and meta-analysis of age, region, and sleep. Sleep Med. 2011;12(2):110-118. 
51. Johns MW. Daytime sleepiness, snoring, and obstructive sleep apnea: the Epworth Sleepiness Scale. Chest. 1993;103(1):30-36.

52. Veenstra G. Social capital, SES and health: an individual-level analysis. Soc Sci Med. 2000;50(5):619-629. doi:10.1016/S0277-9536(99)00307-X

53. Cattell V. Poor people, poor places, and poor health: the mediating role of social networks and social capital. Soc Sci Med. 2001;52(10):1501-1516. doi:10.1016/S0277-9536(00)00259-8

54. Fang J, Wheaton AG, Keenan NL, Greenlund KJ, Perry GS, Croft JB. Association of sleep duration and hypertension among US adults varies by age and sex. $\mathrm{Am} \mathrm{J}$ Hypertens. 2011;25(3):335-341.

55. Grandner MA, Petrov ME, Rattanaumpawan P, Jackson N, Platt A, Patel NP. Sleep symptoms, race/ethnicity, and socioeconomic position. J Clin Sleep Med. 2013;9(9):897-905.

56. Stamatakis KA, Kaplan GA, Roberts RE. Short sleep duration across income, education, and race/ethnic groups: population prevalence and growing disparities during 34 years of follow-up. Ann Epidemiol. 2007;17(12):948-955.

57. Lynch JW, Kaplan GA, Shema SJ. Cumulative impact of sustained economic hardship on physical, cognitive, psychological, and social functioning. $N$ Engl $J$ Med. 1997;337(26):1889-1895.

58. Strawbridge WJ, Wallhagen MI. Self-rated health and mortality over three decades results from a time-dependent covariate analysis. Res Aging. 1999;21(3):402-416.

59. Sarris J, O'Neil A, Coulson CE, Schweitzer I, Berk M. Lifestyle medicine for depression. BMC Psychiatry. 2014;14:107. doi:10.1186/1471-244X-14-107

60. Bliwise DL, Young TB. The Parable of Parabola: What the U-Shaped Curve Can and Cannot Tell Us about Sleep. Sleep. 2007;30(12):1614-1615.

doi:10.1093/sleep/30.12.1614

61. Grandner MA, Drummond SPA. Who are the long sleepers? Towards an understanding of the mortality relationship. Sleep Med Rev. 2007;11(5):341-360. doi:10.1016/j.smrv.2007.03.010 
Table 1. Characteristics of the sample $(N=1,007)$.

Table 1

Demographic charachteristics of the study sample.

\begin{tabular}{|c|c|c|c|c|c|c|c|c|c|c|c|c|c|c|c|c|c|c|c|c|}
\hline \multirow[b]{3}{*}{ Variable } & & \multicolumn{2}{|c|}{ Total } & \multicolumn{4}{|c|}{$\begin{array}{l}\text { Participation in Social or } \\
\text { Community Organizations }\end{array}$} & \multicolumn{4}{|c|}{$\begin{array}{l}\text { Neighborhood } \\
\text { Helping Behavior }\end{array}$} & \multicolumn{3}{|c|}{$\begin{array}{l}\text { Neighborhood } \\
\text { Belonging }\end{array}$} & \multicolumn{3}{|c|}{$\begin{array}{l}\text { Neighborhood } \\
\text { Trust }\end{array}$} & \multicolumn{3}{|c|}{$\begin{array}{l}\text { Neighborhood } \\
\text { Improvement }\end{array}$} \\
\hline & & \multirow[b]{2}{*}{$N$} & \multirow[b]{2}{*}{$\%$} & None & \multirow{2}{*}{$\begin{array}{c}\text { One } \\
\%\end{array}$} & \multirow{2}{*}{$\begin{array}{c}\text { Two+ } \\
\%\end{array}$} & \multirow{2}{*}{$\begin{array}{c}P- \\
\text { Value }\end{array}$} & \multicolumn{3}{|c|}{ Never smetim€ Often } & \multirow{2}{*}{$\begin{array}{c}P- \\
\text { Value }\end{array}$} & \multirow{2}{*}{$\begin{array}{l}\text { No } \\
\% \\
\end{array}$} & \multirow{2}{*}{$\begin{array}{c}\text { Yes } \\
\%\end{array}$} & \multirow{2}{*}{$\begin{array}{c}P- \\
\text { Value }\end{array}$} & \multicolumn{2}{|c|}{ Jisagre؛ Agree } & \multirow{2}{*}{$\begin{array}{c}\text { P- } \\
\text { Value }\end{array}$} & \multirow{2}{*}{$\begin{array}{l}\text { No } \\
\%\end{array}$} & \multirow{2}{*}{$\begin{array}{c}\text { Yes } \\
\%\end{array}$} & \multirow{2}{*}{$\begin{array}{c}P- \\
\text { Value }\end{array}$} \\
\hline & & & & $\%$ & & & & $\%$ & $\%$ & $\%$ & & & & & $\%$ & $\%$ & & & & \\
\hline \multirow{2}{*}{ Age, yrs* } & Mean & & 34.0 & 33.2 & 33.5 & 35.3 & 0.006 & 35.5 & 33.2 & 32.9 & 0.000 & 32.5 & 35 & $<.0001$ & 32.5 & 35 & $<.0001$ & 32.9 & 34.7 & 0.003 \\
\hline & Standard Deviation & & 9.4 & 9.1 & 8.9 & 10.0 & & 10.0 & 9.0 & 8.9 & & 8.5 & 9.9 & & 8.5 & 9.9 & & 8.9 & 9.7 & \\
\hline Sex & Male & 619 & $50.9 \%$ & $39.2 \%$ & $39.4 \%$ & $36.8 \%$ & 0.738 & $35.4 \%$ & $40.7 \%$ & $39.8 \%$ & 0.290 & $40.0 \%$ & $37.6 \%$ & 0.449 & $44.4 \%$ & $35.5 \%$ & 0.449 & $37.0 \%$ & $39.5 \%$ & 0.432 \\
\hline & Female & 597 & $49.1 \%$ & $60.8 \%$ & $60.6 \%$ & $63.3 \%$ & & $64.6 \%$ & $59.3 \%$ & $60.2 \%$ & & $60.1 \%$ & $62.4 \%$ & & $55.6 \%$ & $64.6 \%$ & & $63.0 \%$ & $60.5 \%$ & \\
\hline Race/Ethnicity & Non-Hispanic White & 251 & $58.1 \%$ & $61.9 \%$ & $63.9 \%$ & $53.6 \%$ & 0.113 & $58.7 \%$ & $63.0 \%$ & $55.6 \%$ & 0.467 & $59.0 \%$ & $59.9 \%$ & 0.512 & $46.2 \%$ & $66.5 \%$ & 0.512 & $60.4 \%$ & $59.0 \%$ & 0.010 \\
\hline & Black/African-American & 46 & $10.6 \%$ & $22.2 \%$ & $21.3 \%$ & $31.0 \%$ & & $27.2 \%$ & $22.6 \%$ & $25.5 \%$ & & $23.8 \%$ & $25.9 \%$ & & $36.9 \%$ & $18.8 \%$ & & $21.1 \%$ & $27.3 \%$ & \\
\hline & Hispanic/Latino & 55 & $12.7 \%$ & $5.5 \%$ & $3.9 \%$ & $3.9 \%$ & & $4.4 \%$ & $3.7 \%$ & $6.2 \%$ & & $5.5 \%$ & $4.0 \%$ & & $6.4 \%$ & $3.6 \%$ & & $4.0 \%$ & $4.9 \%$ & \\
\hline & Asian & 54 & $12.5 \%$ & $5.5 \%$ & $6.1 \%$ & $5.1 \%$ & & $4.4 \%$ & $5.3 \%$ & $7.3 \%$ & & $5.3 \%$ & $5.6 \%$ & & $4.1 \%$ & $6.2 \%$ & & $8.3 \%$ & $3.8 \%$ & \\
\hline & Other/Multiracial & 26 & $6.0 \%$ & $5.0 \%$ & $4.8 \%$ & $6.3 \%$ & & $5.4 \%$ & $5.3 \%$ & $5.4 \%$ & & $6.5 \%$ & $4.6 \%$ & & $6.4 \%$ & $4.9 \%$ & & $6.2 \%$ & $4.9 \%$ & \\
\hline Education & Less than high & 26 & $2.6 \%$ & $3.2 \%$ & $2.2 \%$ & $1.8 \%$ & 0.117 & $2.2 \%$ & $2.7 \%$ & $3.1 \%$ & 0.798 & $3.0 \%$ & $2.3 \%$ & 0.679 & $3.2 \%$ & $2.3 \%$ & 0.679 & $1.6 \%$ & $3.2 \%$ & 0.157 \\
\hline & High School & 106 & $10.5 \%$ & $12.5 \%$ & $8.2 \%$ & $9.3 \%$ & & $12.2 \%$ & $9.3 \%$ & $10.0 \%$ & & $9.7 \%$ & $11.1 \%$ & & $15.9 \%$ & $7.7 \%$ & & $9.6 \%$ & $11.1 \%$ & \\
\hline & Some College & 312 & $31.0 \%$ & $32.2 \%$ & $26.4 \%$ & $32.8 \%$ & & $32.2 \%$ & $31.0 \%$ & $29.3 \%$ & & $29.8 \%$ & $31.8 \%$ & & $38.6 \%$ & $27.0 \%$ & & $29.0 \%$ & $32.2 \%$ & \\
\hline & College & 563 & $55.9 \%$ & $52.2 \%$ & $63.2 \%$ & $56.0 \%$ & & $53.5 \%$ & $57.1 \%$ & $57.5 \%$ & & $57.6 \%$ & $54.8 \%$ & & $42.4 \%$ & $63.0 \%$ & & $59.8 \%$ & $53.6 \%$ & \\
\hline Income & $<\$ 20,000$ & 182 & $18.1 \%$ & $18.6 \%$ & $17.8 \%$ & $17.5 \%$ & 0.036 & $14.1 \%$ & $19.3 \%$ & $22.4 \%$ & 0.002 & $23.3 \%$ & $14.7 \%$ & $<.0001$ & $25.4 \%$ & $14.4 \%$ & $<.0001$ & $18.6 \%$ & $17.9 \%$ & 0.963 \\
\hline & $\$ 20,000-\$ 34,999$ & 232 & $23.0 \%$ & $25.2 \%$ & $22.5 \%$ & $20.5 \%$ & & $19.7 \%$ & $25.4 \%$ & $23.9 \%$ & & $24.1 \%$ & $22.2 \%$ & & $28.0 \%$ & $20.3 \%$ & & $22.6 \%$ & $23.1 \%$ & \\
\hline & $\$ 35,000-\$ 49,999$ & 250 & $24.8 \%$ & $28.6 \%$ & $22.1 \%$ & $21.7 \%$ & & $24.1 \%$ & $23.5 \%$ & $27.4 \%$ & & $25.1 \%$ & $24.5 \%$ & & $24.5 \%$ & $24.9 \%$ & & $23.7 \%$ & $25.4 \%$ & \\
\hline & $\$ 50,000-\$ 74,999$ & 157 & $15.6 \%$ & $12.7 \%$ & $17.3 \%$ & $18.4 \%$ & & $18.9 \%$ & $13.2 \%$ & $14.3 \%$ & & $15.4 \%$ & $15.7 \%$ & & $13.5 \%$ & $16.7 \%$ & & $15.7 \%$ & $15.5 \%$ & \\
\hline & $>\$ 75,000$ & 187 & $18.6 \%$ & $15.0 \%$ & $20.4 \%$ & $22.0 \%$ & & $23.2 \%$ & $18.5 \%$ & $12.0 \%$ & & $12.2 \%$ & $22.9 \%$ & & $8.7 \%$ & $23.8 \%$ & & $19.4 \%$ & $18.1 \%$ & \\
\hline Sleep Duration & Very Short $(\leq 4 \mathrm{~h}$ & 96 & $9.5 \%$ & $10.7 \%$ & $6.1 \%$ & $10.2 \%$ & 0.018 & $10.3 \%$ & $6.4 \%$ & $13.1 \%$ & 0.020 & $10.7 \%$ & $8.8 \%$ & 0.081 & $12.4 \%$ & $8.0 \%$ & 0.081 & $8.5 \%$ & $10.1 \%$ & 0.439 \\
\hline & Short ( 5 to 6 hours) & 381 & $37.8 \%$ & $38.3 \%$ & $33.3 \%$ & $40.4 \%$ & & $34.3 \%$ & $39.4 \%$ & $40.5 \%$ & & $41.7 \%$ & $35.3 \%$ & & $43.2 \%$ & $35.0 \%$ & & $41.0 \%$ & $36.0 \%$ & \\
\hline & Normal ( 7 to 8 hours) & 480 & $47.7 \%$ & $44.4 \%$ & $57.1 \%$ & $45.5 \%$ & & $50.5 \%$ & $50.0 \%$ & $40.2 \%$ & & $43.2 \%$ & $50.7 \%$ & & $40.1 \%$ & $51.7 \%$ & & $45.7 \%$ & $48.8 \%$ & \\
\hline & Long ( $>9$ hours) & 50 & $5.0 \%$ & $6.6 \%$ & $3.5 \%$ & $3.9 \%$ & & $4.9 \%$ & $4.2 \%$ & $6.2 \%$ & & $4.5 \%$ & $5.3 \%$ & & $4.3 \%$ & $5.3 \%$ & & $4.8 \%$ & $5.1 \%$ & \\
\hline Insomnia Symptoms $†$ & None & 350 & $34.8 \%$ & $34.2 \%$ & $35.5 \%$ & $34.9 \%$ & 0.105 & $34.9 \%$ & $36.2 \%$ & $32.4 \%$ & 0.112 & $29.8 \%$ & $38.1 \%$ & 0.010 & $27.4 \%$ & $38.6 \%$ & 0.010 & $31.7 \%$ & $36.6 \%$ & 0.140 \\
\hline & Mild & 389 & $38.6 \%$ & $36.1 \%$ & $44.2 \%$ & $38.3 \%$ & & $41.9 \%$ & $37.8 \%$ & $35.1 \%$ & & $39.5 \%$ & $38.1 \%$ & & $38.9 \%$ & $38.5 \%$ & & $38.6 \%$ & $38.7 \%$ & \\
\hline & Moderate & 268 & $26.6 \%$ & $29.7 \%$ & $20.4 \%$ & $26.8 \%$ & & $23.2 \%$ & $25.9 \%$ & $32.4 \%$ & & $30.8 \%$ & $23.8 \%$ & & $33.7 \%$ & $22.9 \%$ & & $29.8 \%$ & $24.7 \%$ & \\
\hline Daytime Sleepiness* & Mean & & 7.8 & 8.1 & 7.5 & 7.7 & 0.208 & 7.54 & 7.74 & 8.43 & 0.056 & 8.4 & 7.5 & 0.002 & 8.42 & 7.46 & 0.002 & 7.8 & 7.9 & 0.960 \\
\hline & Standard Deviation & & 4.7 & 4.6 & 4.3 & 4.7 & & 4.7 & 4.4 & 4.8 & & 4.9 & 4.4 & & 4.8 & 4.4 & & 4.6 & 4.6 & \\
\hline
\end{tabular}

* Data displayed in mean, standard deviation.

* Responses collected on a scale from 1 to 7.

† Insomnia was scored as "none" for participants who scored 0 to 7 on the ISI, as "mild" for participants who scored 8 to 14 on the ISI, and "moderate-severe" for participants who scored $15-28$ 
Table 2. Binomial logistic regression, single and combined models, examining the relationship between sleep (duration, insomnia, and sleepiness) and participation in social or community organizations ( 0 and 1 or more organizations), adjusting for age, sex, race/ethnicity, education, and income $(\mathrm{N}=1,007)$.

\begin{tabular}{|c|c|c|c|c|c|c|c|c|}
\hline \multirow{4}{*}{\multicolumn{2}{|c|}{ Variables (Single/Combined Models) }} & \multicolumn{7}{|c|}{$\begin{array}{l}\text { Participation in Social or Community Organizations } \\
\text { (Comparison: No Participation)* }\end{array}$} \\
\hline & & \multirow[b]{3}{*}{$O R$} & \multirow{2}{*}{\multicolumn{2}{|c|}{$\frac{\text { One Membership }}{\text { C.I. }}$}} & \multirow{3}{*}{$\begin{array}{c}P- \\
\text { Value }\end{array}$} & \multicolumn{3}{|c|}{ Two+ Memberships } \\
\hline & & & & & & & C.I. & $P_{-}$ \\
\hline & & & Lower & Upper & & OR & Lower Upper & Value \\
\hline \multicolumn{9}{|c|}{ Separate Models } \\
\hline \multirow[t]{4}{*}{ Sleep Duration } & Very Short ( $\leq 4 \mathrm{hrs})$ & $\underline{0.5}$ & $\underline{0.3}$ & $\underline{1.0}$ & $\underline{0.044}$ & $\underline{0.9}$ & $\underline{0.5} \quad \underline{1.5}$ & $\underline{0.712}$ \\
\hline & Short (5-6hrs) & 0.7 & $\overline{0.5}$ & $\overline{1.0}$ & $\overline{0.067}$ & $\overline{1.0}$ & $\overline{0.7}$ & $\overline{0.976}$ \\
\hline & Recommended duration (7-8hrs) & 1.00 & & eference & & & Reference & \\
\hline & Long (>9hrs) & $\overline{0.4}$ & $\underline{0.2}$ & $\underline{0.9}$ & $\underline{0.031}$ & $\underline{0.6}$ & $\underline{0.3} \quad \underline{1.2}$ & $\underline{0.133}$ \\
\hline \multirow[t]{3}{*}{ Insomnia $^{\dagger}$} & None & $\overline{1.00}$ & & eference & & & $\overline{\text { Reference }}$ & \\
\hline & Mild & $\overline{1.3}$ & 0.9 & $\underline{1.8}$ & $\underline{0.235}$ & 1.1 & $0.8 \quad 1.5$ & $\underline{0.624}$ \\
\hline & Moderate-Severe & $\overline{0.7}$ & $\overline{0.5}$ & $\overline{1.1}$ & $\overline{0.169}$ & $\overline{0.9}$ & $\overline{0.6}$ & $\overline{0.751}$ \\
\hline Sleepiness $\ddagger$ & High & $\underline{0.8}$ & $\underline{0.6}$ & $\underline{1.2}$ & $\underline{0.320}$ & $\underline{0.8}$ & $\underline{0.6}$ & $\underline{0.163}$ \\
\hline \multicolumn{9}{|l|}{ Combined Models } \\
\hline \multirow[t]{4}{*}{ Sleep Duration } & Very Short ( $\leq 4 \mathrm{hrs}$ ) & $\underline{0.5}$ & $\underline{0.3}$ & 1.1 & $\underline{0.101}$ & 1.0 & $\underline{0.5}$ & $\underline{0.895}$ \\
\hline & Short (5-6hrs) & $\underline{0.7}$ & $\underline{0.5}$ & $\underline{1.0}$ & $\underline{0.076}$ & $\underline{1.0}$ & $\overline{0.7}, \overline{1.4}$ & $\underline{0.957}$ \\
\hline & Recommended duration (7-8hrs) & $\underline{1.00}$ & & eference & & & Reference & \\
\hline & Long (>9hrs) & $\overline{0.4}$ & $\underline{0.2}$ & $\underline{0.9}$ & $\underline{0.031}$ & $\underline{0.6}$ & $\underline{0.3} \quad \underline{1.2}$ & $\underline{0.131}$ \\
\hline \multirow[t]{3}{*}{ Insomnia $^{\dagger}$} & None & $\overline{1.00}$ & & eference & & & $\overline{\text { Reference }}$ & \\
\hline & Mild & $\overline{1.4}$ & 1.0 & 2.1 & $\underline{0.086}$ & 1.1 & $\underline{0.8} \quad \underline{1.6}$ & $\underline{0.513}$ \\
\hline & Moderate-Severe & $\overline{0.9}$ & $\overline{0.6}$ & 1.5 & 0.778 & $\overline{1.0}$ & $\overline{0.7}$ & 0.929 \\
\hline Sleepiness $\ddagger$ & High & $\underline{0.9}$ & 0.6 & 1.3 & 0.568 & $\underline{0.8}$ & 0.6 & 0.161 \\
\hline
\end{tabular}

†Insomnia was scored as "none" for participants who scored 0 to 7 on the ISI, as "mild" for participants who scored 8 to 14 on the ISI, and "moderate-severe" for participants who scored 15 to 28.

¥Sleepiness was scored as "high" for participants who scored 10 or higher on the ESS. 
Table 3. Multinomial logistic regression, single and combined models, examining the relationship between sleep (duration, insomnia, and sleepiness) and neighbor helping behavior (often/always, sometimes, and rarely/never), adjusting for age, sex, race/ethnicity, income, and education $(\mathrm{N}=1,007)$.

\begin{tabular}{|c|c|c|c|c|c|c|c|c|c|}
\hline \multirow{4}{*}{\multicolumn{2}{|c|}{ Variables (Single/Combined Models) }} & \multicolumn{8}{|c|}{$\begin{array}{l}\text { Neighborhood Helping Behavior } \\
\text { (Comparison: Often/Always)* }\end{array}$} \\
\hline & & \multicolumn{4}{|c|}{ Sometimes } & \multicolumn{4}{|c|}{ Rarely/Never } \\
\hline & & \multirow[b]{2}{*}{ OR } & \multicolumn{2}{|c|}{ C.I. } & \multirow{2}{*}{$\begin{array}{c}P- \\
\text { Value }\end{array}$} & \multirow[b]{2}{*}{$O R$} & \multicolumn{2}{|c|}{ C.I. } & \multirow{2}{*}{$\begin{array}{c}P- \\
\text { Value }\end{array}$} \\
\hline & & & Lower & Upper & & & Lower & Upper & \\
\hline \multicolumn{10}{|c|}{ Separate Models } \\
\hline \multirow[t]{4}{*}{ Sleep Duration } & Very Short ( $\leq 4 \mathrm{hrs})$ & $\underline{0.7}$ & $\underline{0.3}$ & 1.2 & $\underline{0.24}$ & 1.8 & 1.0 & $\underline{3.2}$ & $\underline{0.039}$ \\
\hline & Short (5-6hrs) & $\overline{1.2}$ & 0.9 & $\overline{1.7}$ & $\overline{0.18}$ & $\overline{1.6}$ & $\overline{1.1}$ & $\overline{2.3}$ & 0.009 \\
\hline & Recommended duration (7-8hrs) & $\overline{1.0}$ & & & & ferenc & & & \\
\hline & Long (>9hrs) & $\underline{0.8}$ & $\underline{0.3}$ & 1.6 & $\underline{0.537}$ & 1.4 & $\underline{0.6}$ & $\underline{2.9}$ & $\underline{0.355}$ \\
\hline \multirow[t]{3}{*}{ Insomnia $^{\dagger}$} & None & $\overline{1.0}$ & & & & ferenc & & & \\
\hline & Mild & $\overline{0.8}$ & 0.6 & 1.2 & 0.356 & 0.8 & 0.6 & 1.3 & 0.562 \\
\hline & Moderate-Severe & $\overline{1.1}$ & 0.8 & $\overline{1.6}$ & 0.545 & $\overline{1.6}$ & 1.0 & $\overline{2.5}$ & 0.026 \\
\hline Sleepinessł & High & 1.0 & 1.0 & $\overline{1.0}$ & $\underline{0.495}$ & 1.0 & $\underline{1.0}$ & $\underline{1.1}$ & 0.023 \\
\hline \multicolumn{10}{|l|}{ Combined Model } \\
\hline \multirow{4}{*}{ Sleep Duration } & Very Short ( $\leq 4 \mathrm{hrs})$ & $\underline{0.6}$ & $\underline{0.3}$ & 1.2 & $\underline{0.160}$ & 1.5 & $\underline{0.9}$ & 2.8 & $\underline{0.221}$ \\
\hline & Short (5-6hrs) & $\overline{1.2}$ & 0.9 & $\overline{1.7}$ & 0.239 & $\overline{1.6}$ & $\overline{1.1}$ & $\overline{2.3}$ & 0.026 \\
\hline & Recommended duration (7-8hrs) & 1.0 & & & & ferenc & & & \\
\hline & Long (>9hrs) & $\underline{0.8}$ & $\underline{0.4}$ & $\underline{1.6}$ & $\underline{0.531}$ & 1.4 & $\underline{0.7}$ & $\underline{2.9}$ & $\underline{0.379}$ \\
\hline \multirow[t]{3}{*}{ Insomnia $^{\dagger}$} & None & 1.0 & & & & ferenc & & & \\
\hline & Mild & 0.8 & 0.6 & 1.2 & 0.239 & 0.8 & 0.5 & 1.1 & 0.170 \\
\hline & Moderate-Severe & $\overline{1.1}$ & 0.7 & $\overline{1.8}$ & 0.651 & $\overline{1.2}$ & 0.7 & 2.0 & 0.492 \\
\hline Sleepiness $\ddagger$ & High & 1.0 & 1.0 & $\overline{1.1}$ & 0.597 & $\overline{1.0}$ & 1.0 & $\overline{1.1}$ & 0.158 \\
\hline
\end{tabular}

†Insomnia was scored as "none" for participants who scored 0 to 7 on the ISI, as "mild" for participants who scored 8 to 14 on the ISI, and "moderate-severe" for participants who scored 15 to 28.

¥Sleepiness was scored as "high" for participants who scored 10 or higher on the ESS. 
Table 4. Binomial logistic regression, single and combined models, examining the relationship between sleep (duration,

2 insomnia, and sleepiness) and neighborhood belonging, trust, and improvement adjusting for age, sex, race/ethnicity,

3 education, and income $(\mathrm{N}=1,007)$.

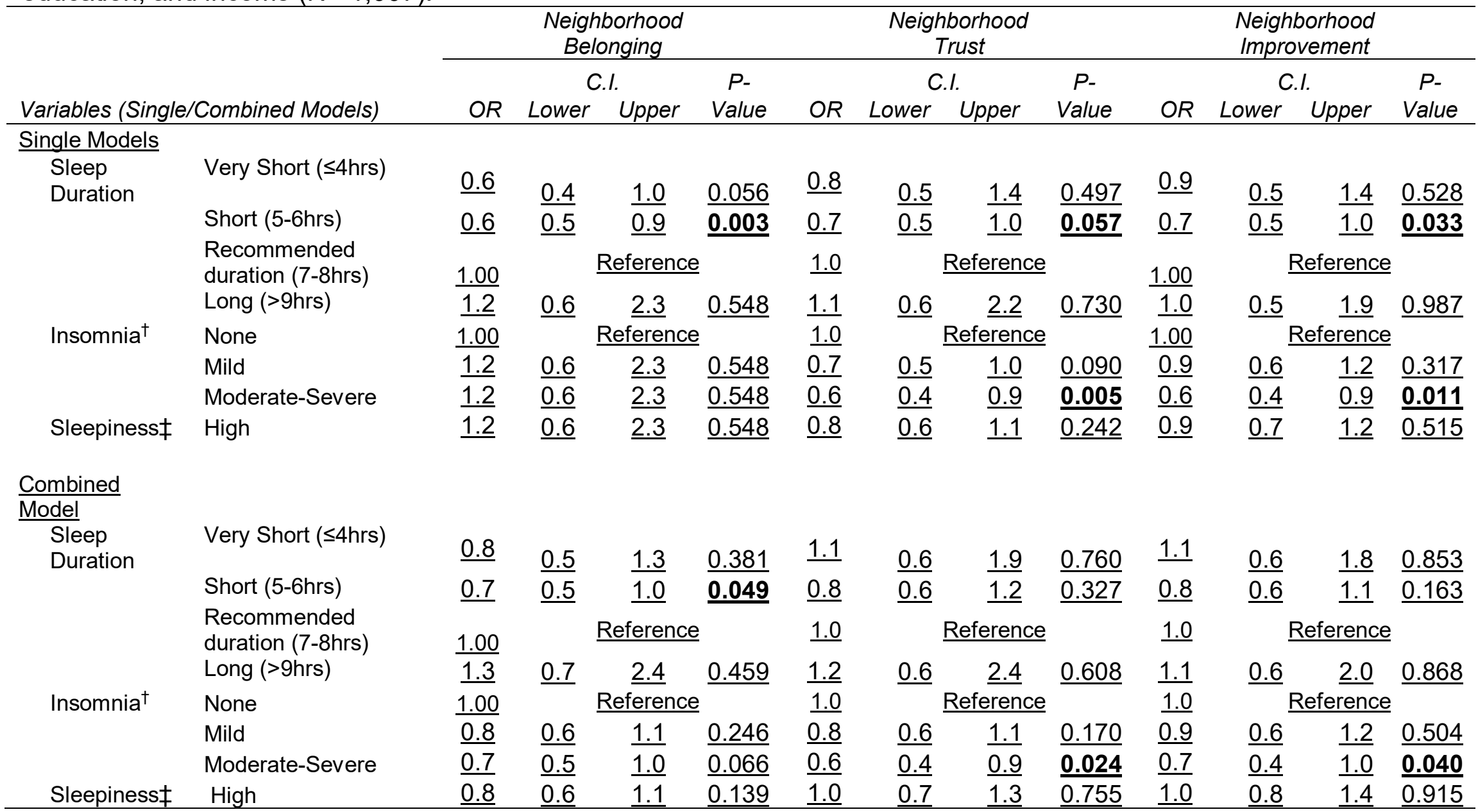

4 "Reference groups include "disagree" for belonging, "disagree" for trust, and "no" for improvement.

5 tInsomnia was scored as "none" for participants who scored 0 to 7 on the ISI, as "mild" for participants who scored 8 to 14 on the ISI,

6 and "moderate-severe" for participants who scored 15 to 28.

$7 \quad \ddagger$ Sleepiness was scored as "high" for participants who scored 10 or higher on the ESS. 